\title{
RCM of tripeptide dienes containing a chiral vinylcyclopropane moiety - Impact of different Ru-based catalysts on the stereochemical integrity of the macrocyclic products.
}

\author{
Martin Poirier, Norman Aubry, Colette Boucher, Jean-Marie Ferland, Steven R. LaPlante \\ and Youla S. Tsantrizos* \\ Department of Chemistry, Boehringer Ingelheim (Canada) Ltd., Research and \\ Development, 2100 Cunard Street, Laval, Quebec, Canada
}

$\begin{array}{ll}\text { RCM Reaction Conditions } & \text { pS2 }\end{array}$

General Protocol for HPLC Analysis, Purification and Characterization $\quad$ pS2

$\begin{array}{ll}\text { NMR Methods } & \text { pS3 }\end{array}$

${ }^{1} \mathrm{H}$ NMR spectrum of acid analog of compound $\mathbf{3 b} \quad$ pS4

${ }^{1} \mathrm{H}$ NMR, P1 H $\beta$ decoupling of acid analog of compound $\mathbf{3 b} \quad$ pS4

ROESY spectrum of acid analog of compound $\mathbf{3 b} \quad$ pS5

ROESY spectrum of acid analog of compound 3c pS6

$\begin{array}{ll}{ }^{1} \mathrm{H} \text { NMR spectrum of acid analog of compound } E-4 d & \text { pS7 }\end{array}$

${ }^{1} \mathrm{H}$ NMR, P1He decoupling of acid analog of compound E-4d $\quad$ pS7

${ }^{1} \mathrm{H}$ NMR spectrum of acid analog of compound $E-4 \mathbf{e} \quad$ pS8

ROESY spectrum of acid analog of compound $E-\mathbf{4 e} \quad$ pS9

${ }^{1} \mathrm{H}$ NMR spectrum of acid analog of compound 5e $\quad$ pS10

ROESY spectrum of acid analog of compound 5e pS11 
${ }^{1} \mathrm{H}$ NMR spectrum of diene 9

pS12

${ }^{1} \mathrm{H}$ NMR spectrum of compound $\mathbf{1 0}$

pS13

${ }^{13} \mathrm{C}$ NMR spectrum of compound $\mathbf{1 0}$

$\mathrm{pS} 13$

Section of the ROESY spectrum of compound $\mathbf{1 0}$

$\mathrm{pS} 14$

RCM Reaction Conditions: Each diene starting material was dissolved in the solvent indicated in Tables 1 and 2 , at a concentration of $0.01 \mathrm{M}$, and the solution was deoxygenated by bubbling argon $(\sim 1 \mathrm{~h}$ for a volume of $500 \mathrm{~mL})$. The Ru-based catalyst was dissolved in a small amount of degassed solvent and added to the reaction mixture (mmol\% as indicated in Tables 1 and 2). The reaction was stirred at the temperature indicated (Tables 1 and 2) and complete conversion of each diene to products was monitored by analytical HPLC using a $\mathrm{C}_{18}$ reversed phase column. For each RCM reaction, the presence (or absence) of multiple macrocyclic products, unreacted starting material, or dimmers/polymers of the acyclic diene, was monitored by analytical $\mathrm{C}_{18}$ reversed phase LC-MS, analytical $\mathrm{C}_{18}$ reversed phase HPLC and Chiral HPLC as indicated below.

General Protocol for HPLC Analysis, Purification and Characterization of Products: After each RCM reaction, the crude reaction mixture was concentrated to minimum volume under reduced pressure and filtered through a short pad of silica gel, eluting first with $\mathrm{CH}_{2} \mathrm{Cl}_{2}$ (to remove the catalyst) and then with EtOAc to isolate the mixture of macrocyclic or acyclic ester products. Model compounds, such as those shown in Table 2, were further purified by flash column chromatography (conditions indicated below) and semi-preparative $\mathrm{C}_{18}$ reversed phase HPLC. HPLC purification was carried out using a solvent gradient from 5\% 
to $100 \%$ aqueous $\mathrm{CH}_{3} \mathrm{CN}$ (all solvents contained $0.06 \%$ TFA). The final homogeneity of each inhibitor was determined by analytical $\mathrm{C}_{18}$ reversed phase HPLC using a Vydac $\mathrm{C}_{18}$ reversed phase column $(0.46 \times 12.5 \mathrm{~cm}$ I.D., $5 \mu \mathrm{m}, 300 \AA)$ and a 35 min linear gradient from 0 to $100 \%$ aqueous $\mathrm{CH}_{3} \mathrm{CN}$ (all solvents contained $0.06 \%$ TFA) at a flow rate of $1.5 \mathrm{~mL} / \mathrm{min}$ with the UV detector set at $\lambda=220 \mathrm{~nm}$. Aliquots of the RCM reaction mixtures, crude products (i.e. reactions shown in Tables 1 and 2) and final purified products were also analyzed by chiral HPLC using a ChiralCel OJ-R column (particle side $=5 \mu \mathrm{m}$, i.d. $=4.6 \times 150$ $\mathrm{mm}$ ). The UV detector was set at $\lambda=205 \mathrm{~nm}$, and the compound(s) were eluted from the column using a solvent gradient from $0.06 \%$ aqueous TFA to $\mathrm{CH}_{3} \mathrm{CN}$ containing $0.06 \%$ TFA.

NMR Methods: ${ }^{1} \mathrm{H}$ NMR spectra of all synthetic intermediates and products were obtained at $27^{\circ} \mathrm{C}$ using a $400 \mathrm{MHz}$ spectrometer and the chemical shifts are given in ppm, referenced to the internal deuterated solvent. Two-dimensional (2D) double quantum-filtered COSY (DQF-COSY), and ROESY spectra were acquired using standard pulse sequences. The ROESY experiments were recorded with a $200 \mathrm{~ms}$ spin-lock period. The $2 \mathrm{D}$ data sets were typically acquired with 2048 points in $t_{2}, 350-512$ points in $t_{1}$ and $32-64$ scans. The data were processed and analyzed using XWinNMR and WinNMR software (Bruker Canada, Milton, Ontario) and Felix software (Molecular Simulations Inc., San Diego, CA). Shifted sine-bell apodization and zero-filling $(2048 \times 1048$ real points $)$ were applied prior to Fourier transformation, and subsequent baseline corrections were applied in both dimensions. 


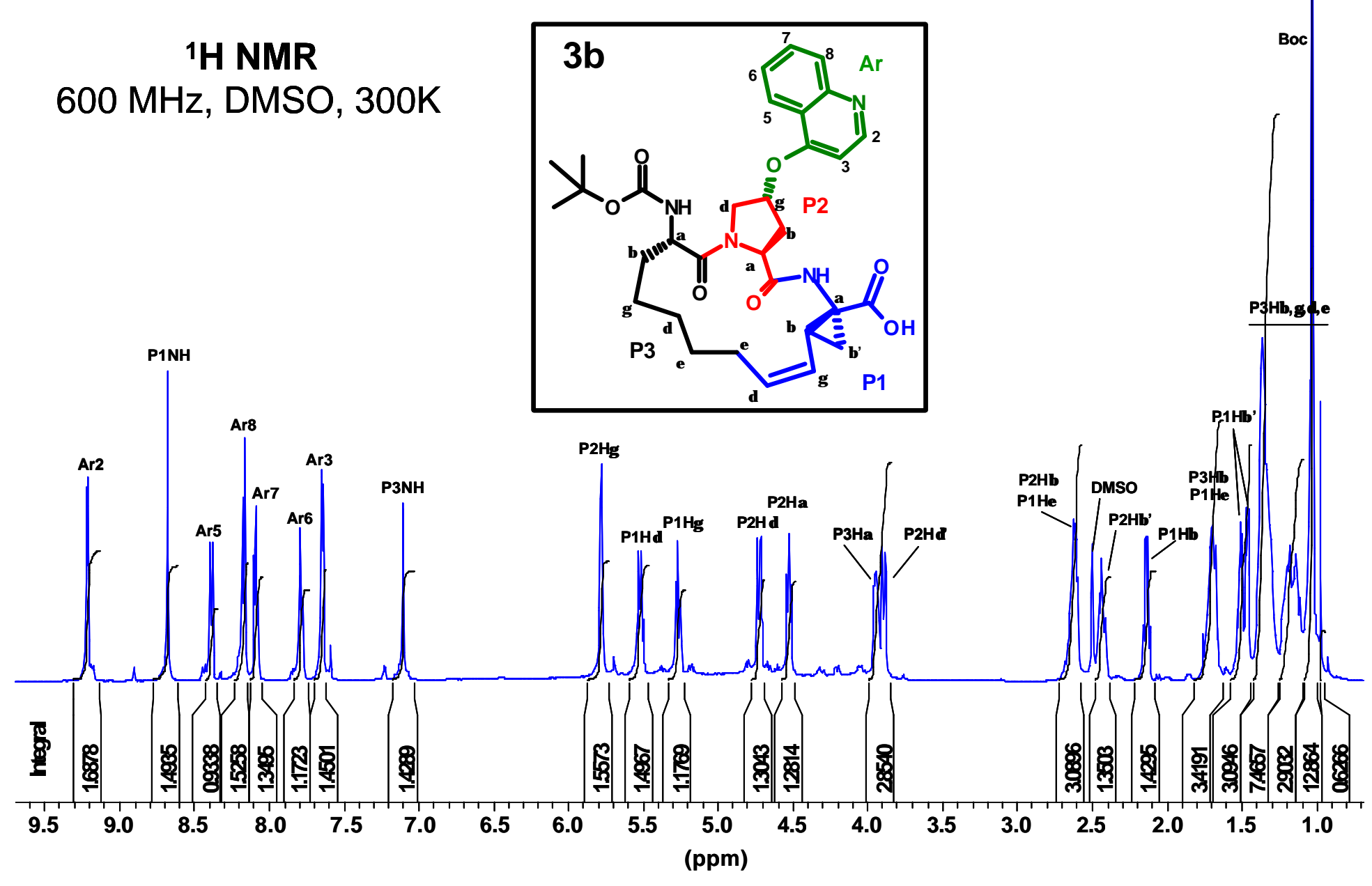

\begin{tabular}{lcccl}
\hline Identifier & Chem. Shift [ppm] & $\mathrm{J}[\mathrm{Hz}]$ & Multiplicity & Connections \\
\hline 1 & 5.2762 & 10.4904 & 2 & $\mathrm{~J}(1,0)$ \\
\hline
\end{tabular}

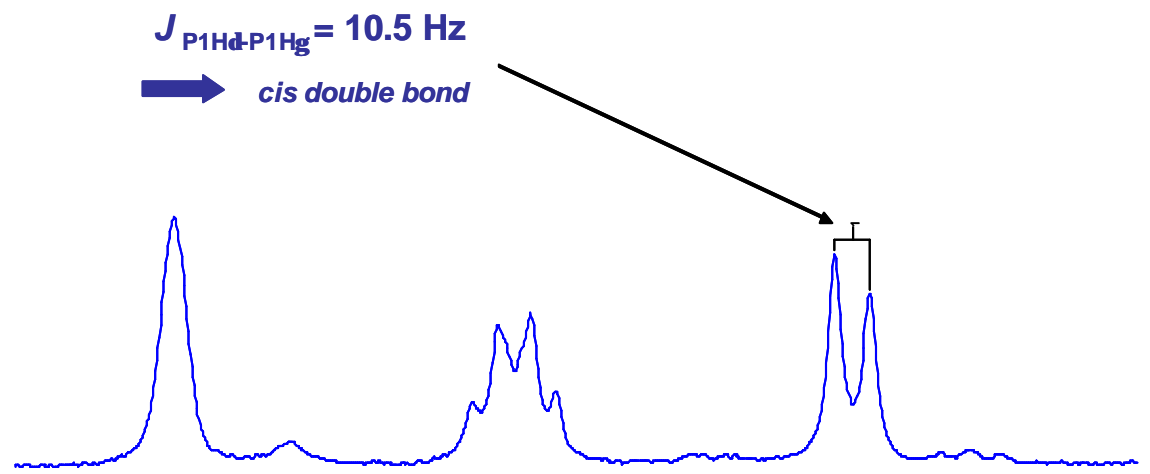




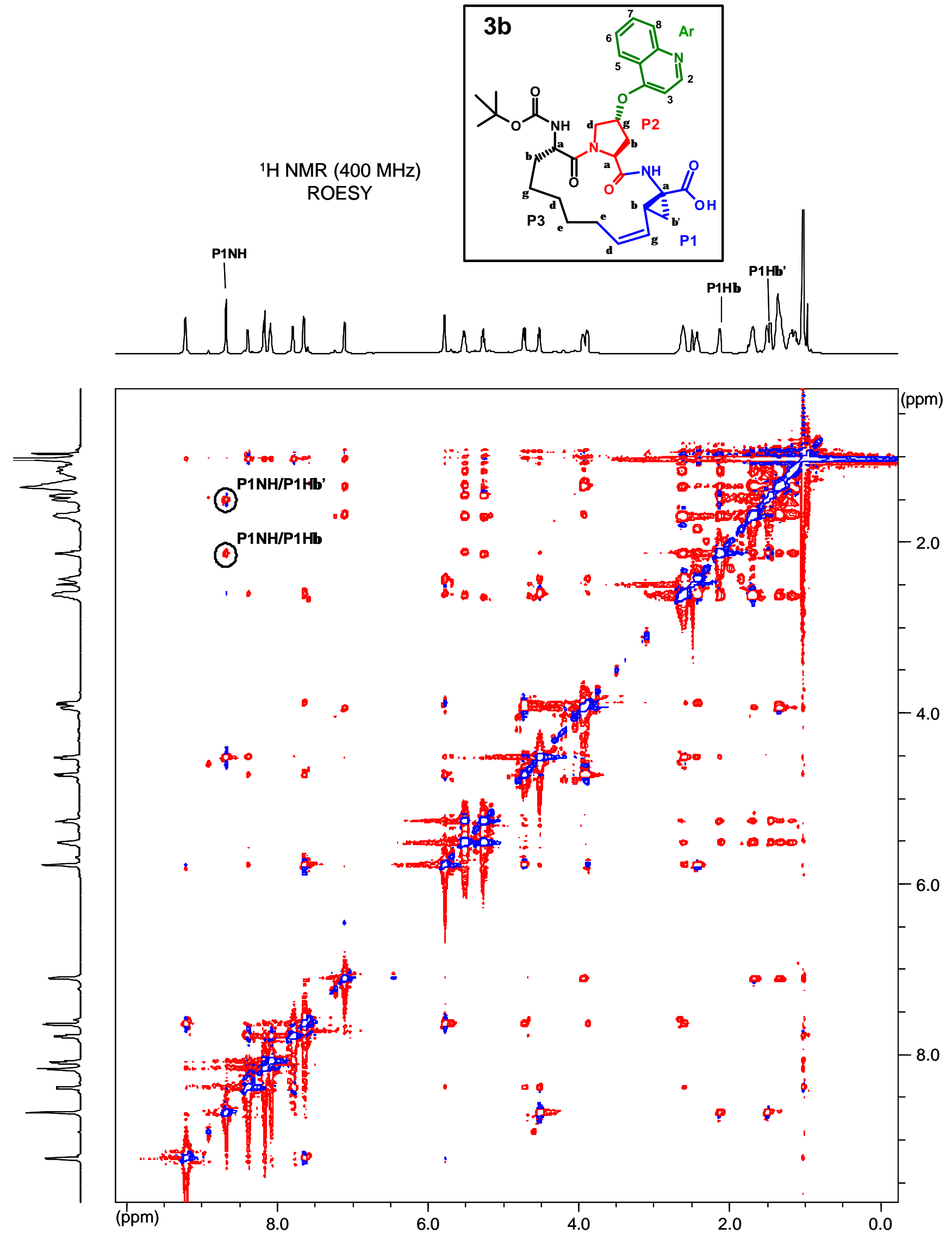




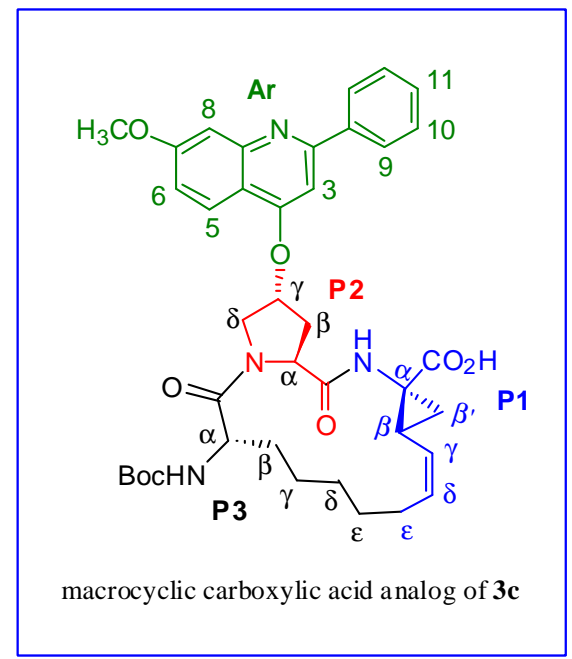

Determination of stereochemistry at C $\beta$ of the P1 vinyl ACCA moiety from the ROESY NMR data

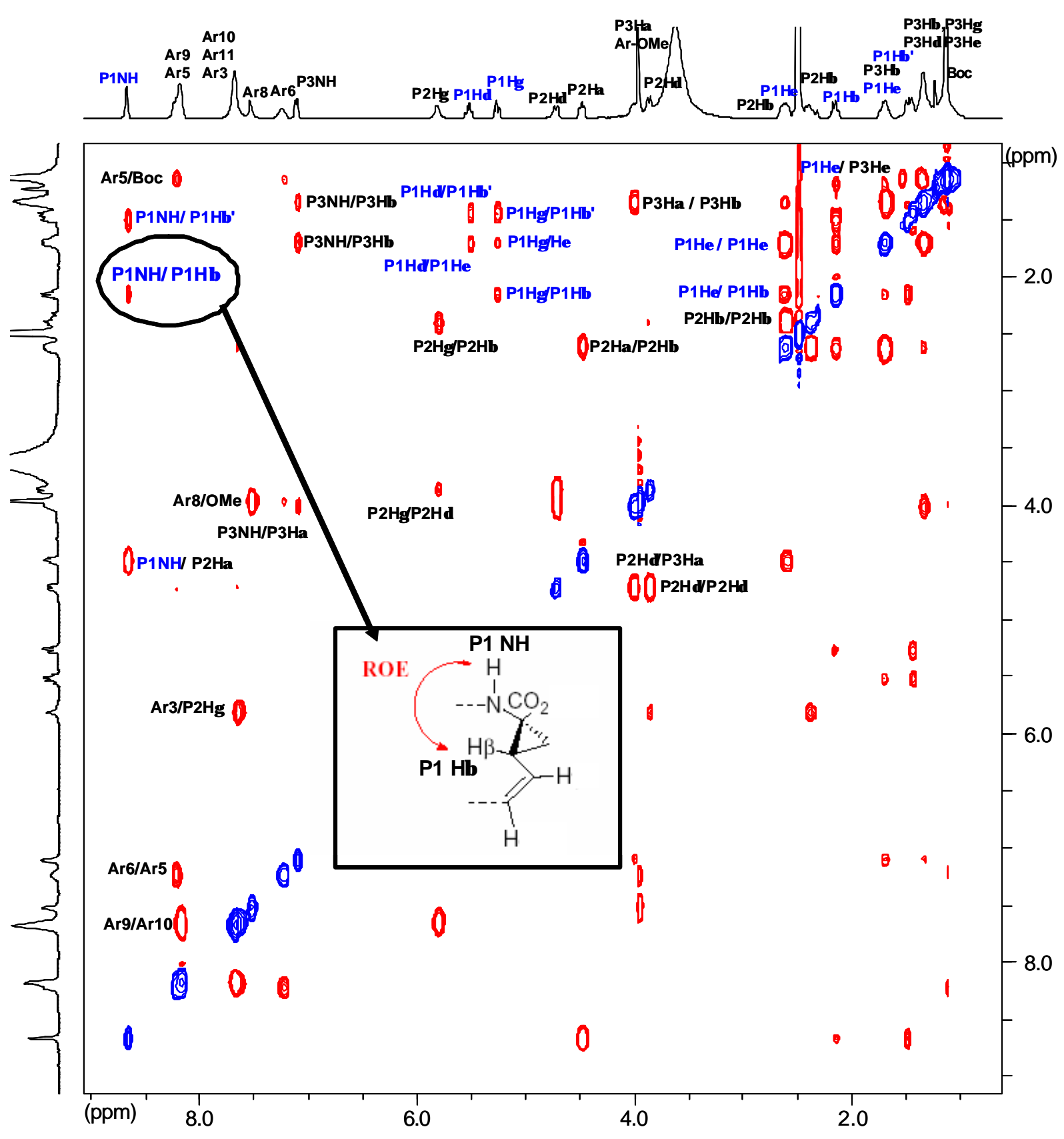



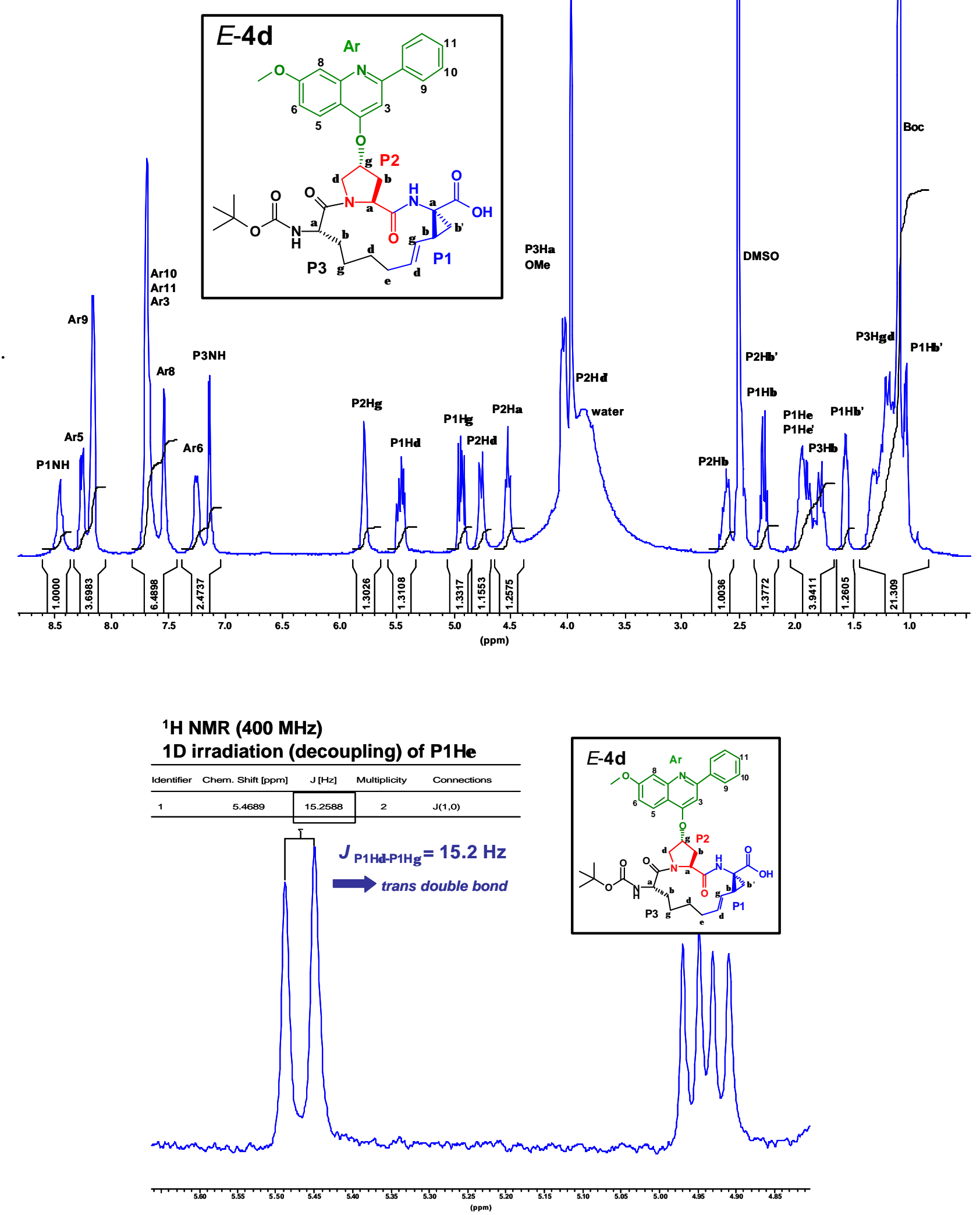


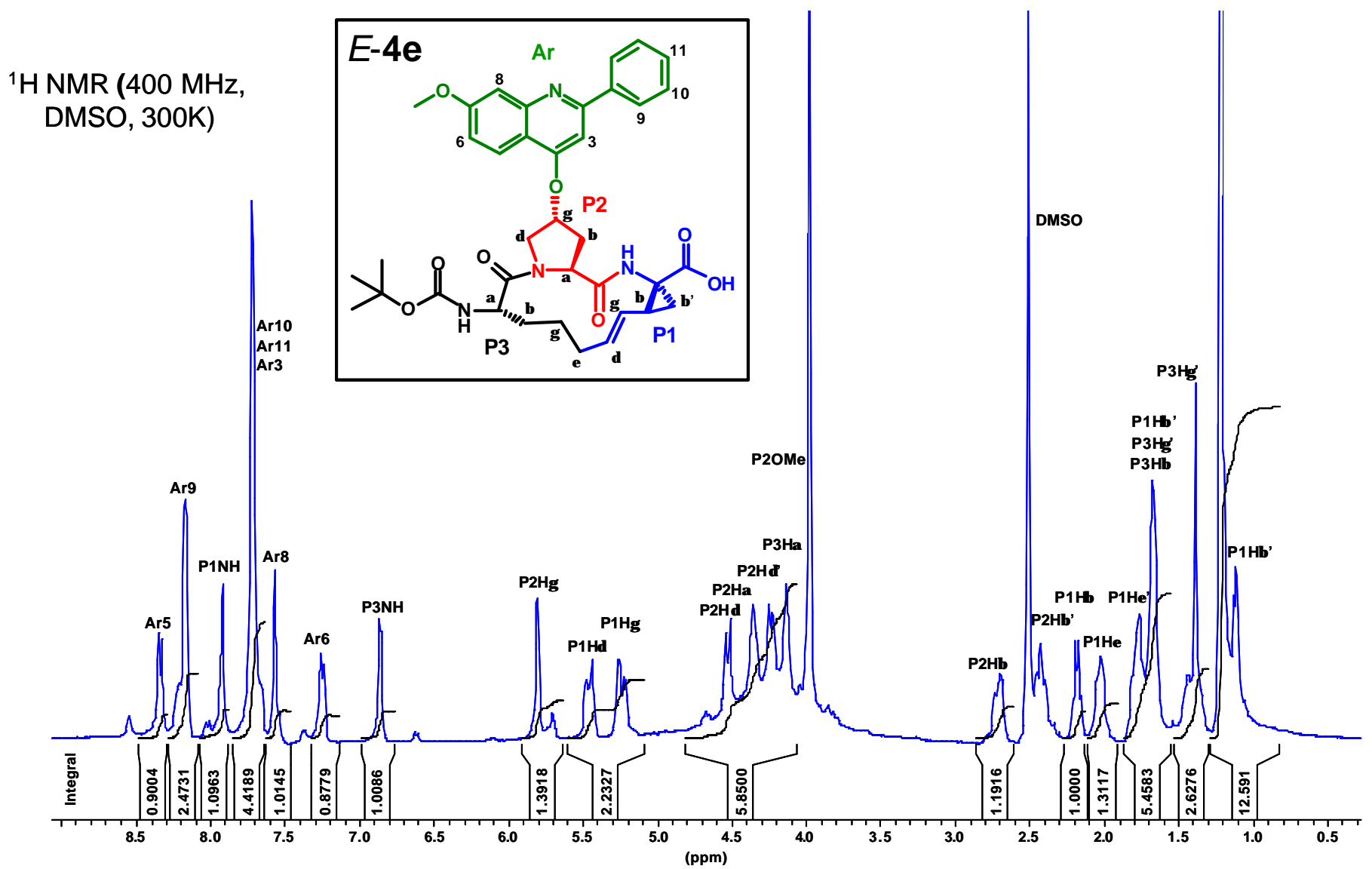


1H NMR (400 MHz) ROESY
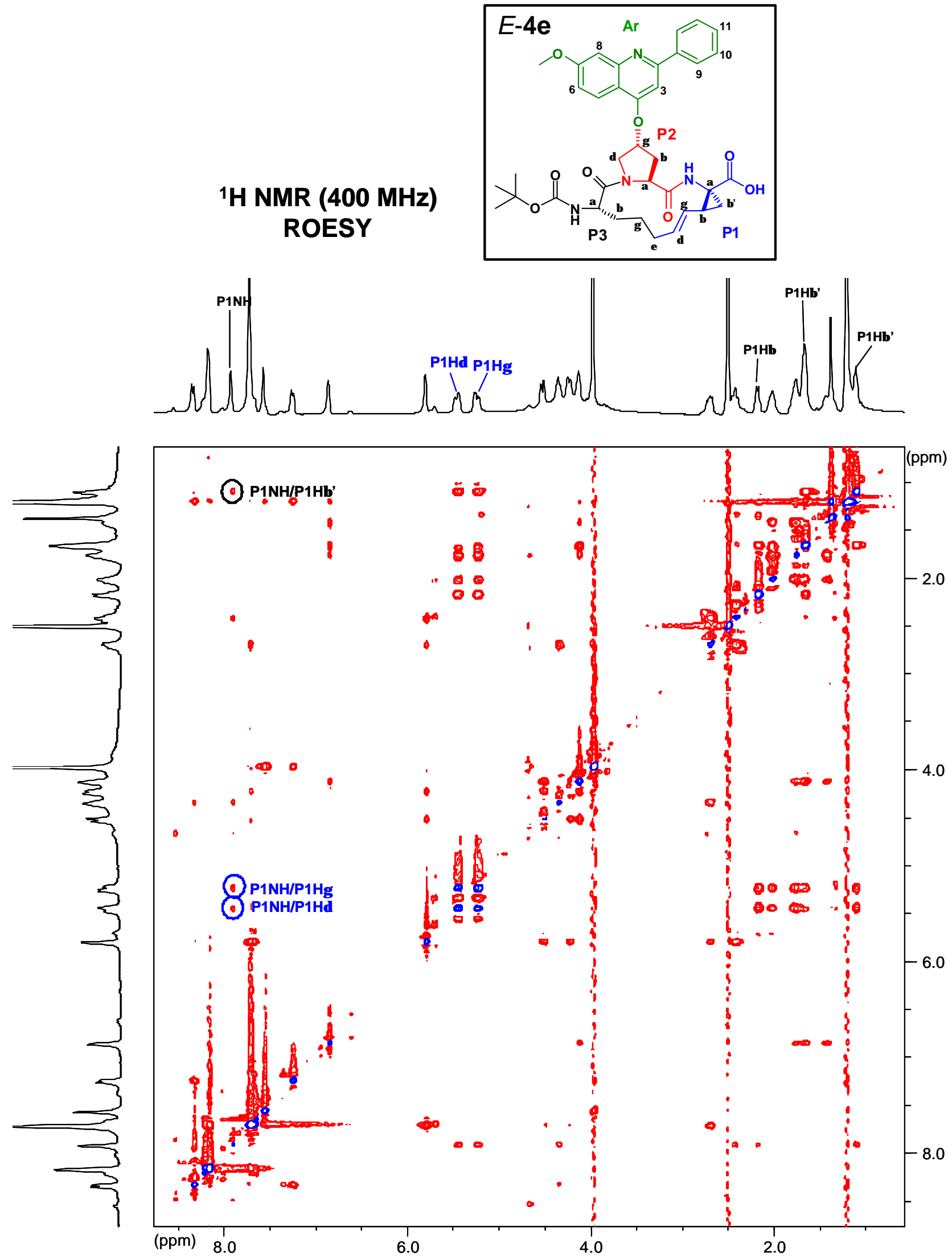


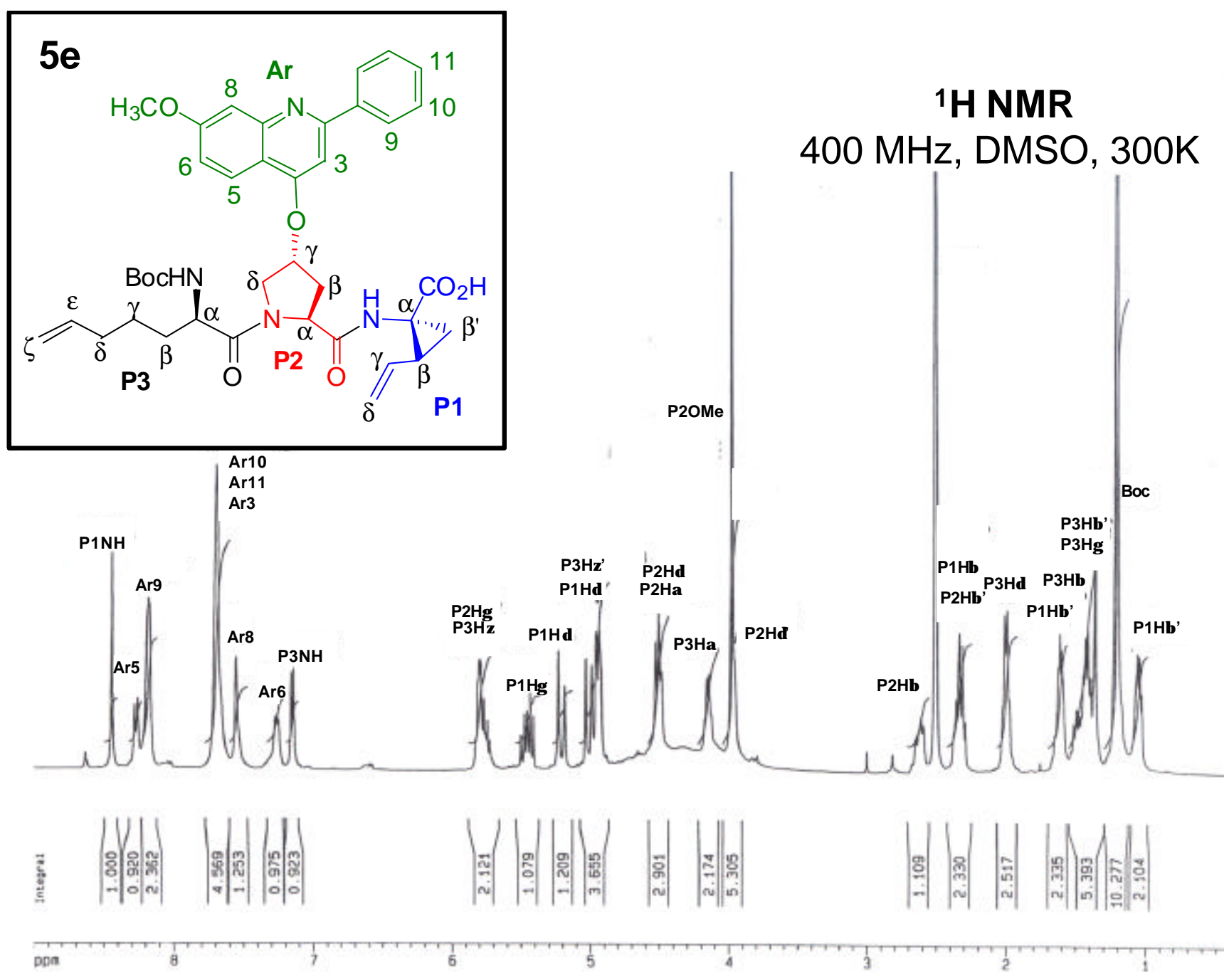



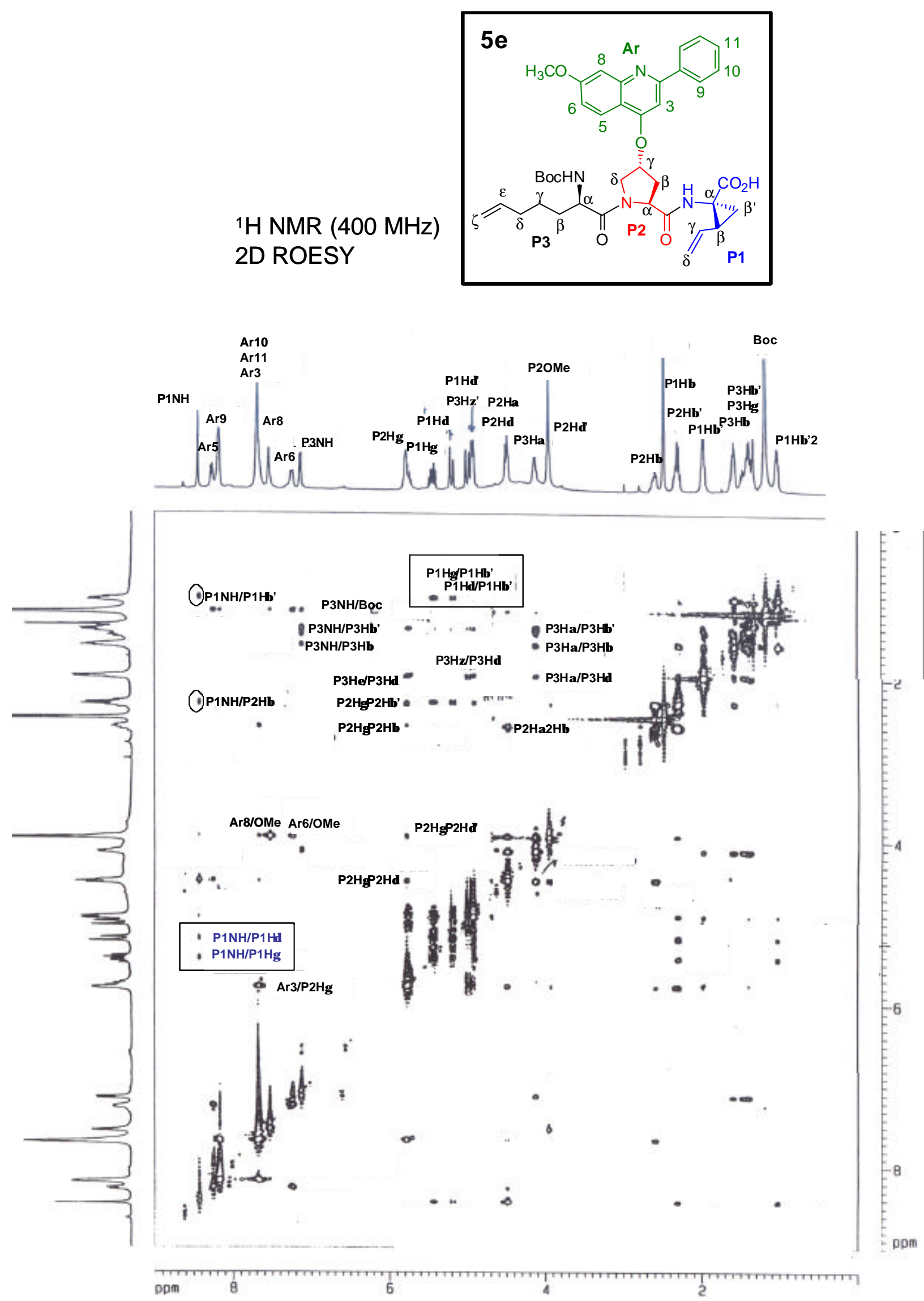


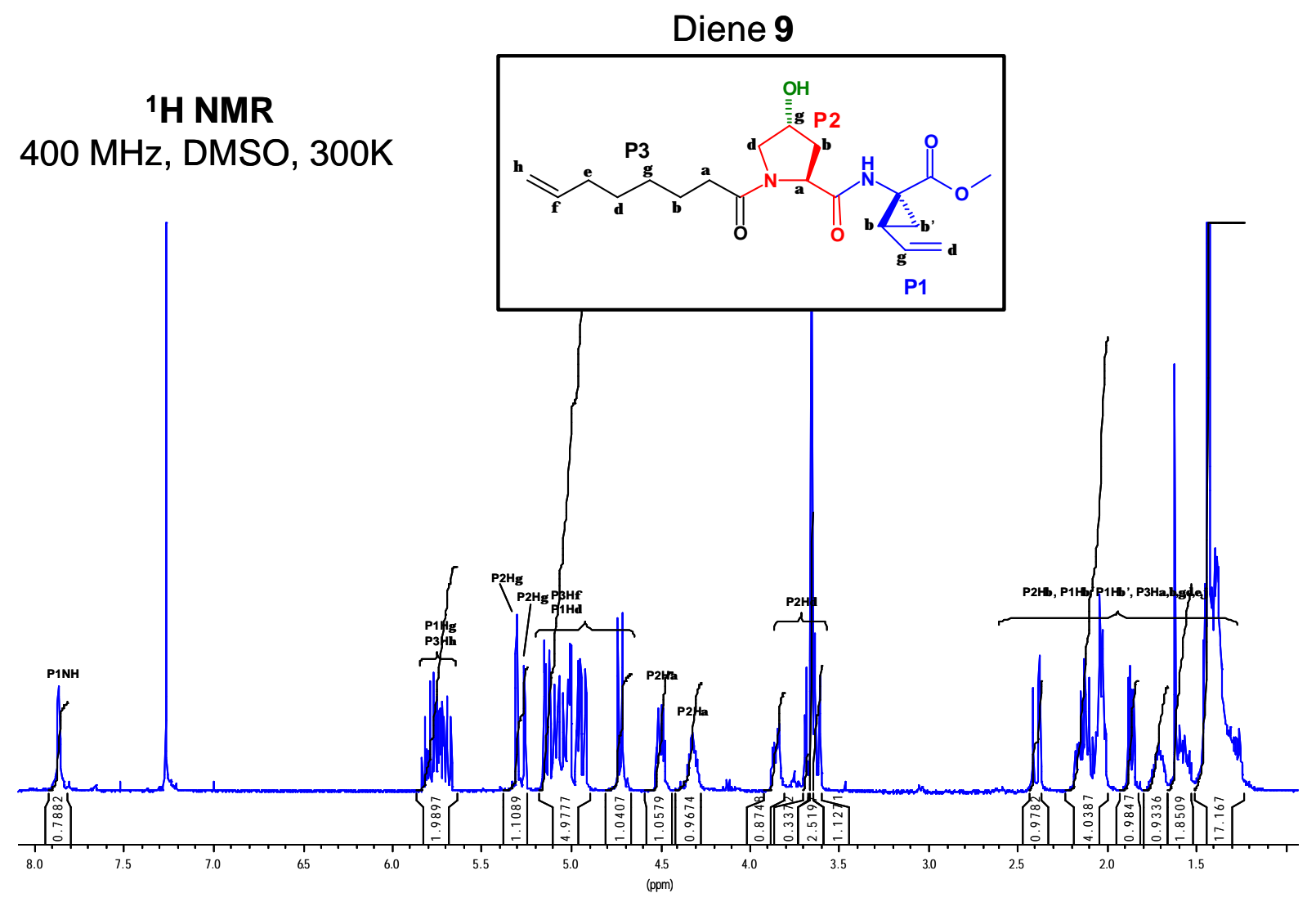



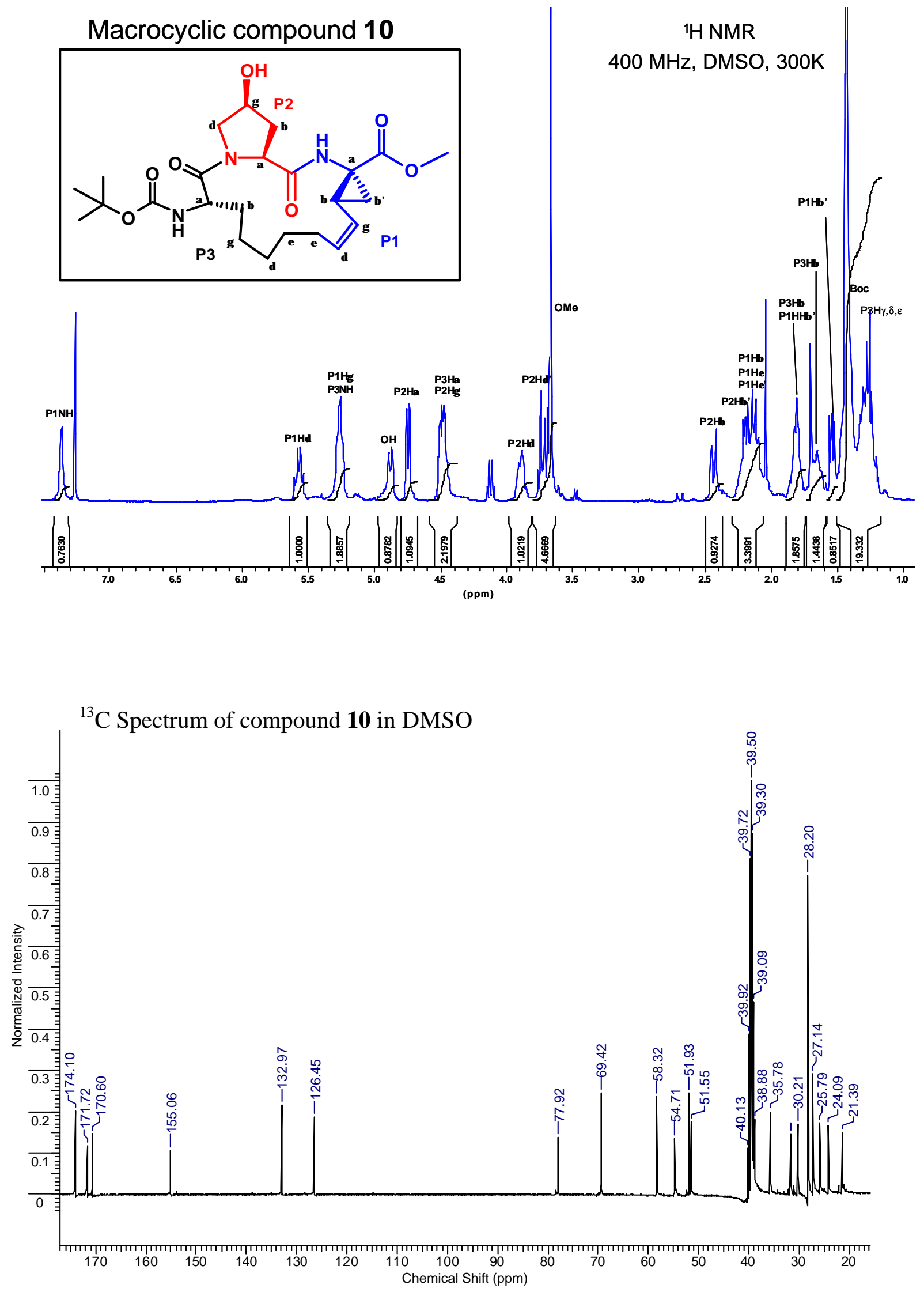


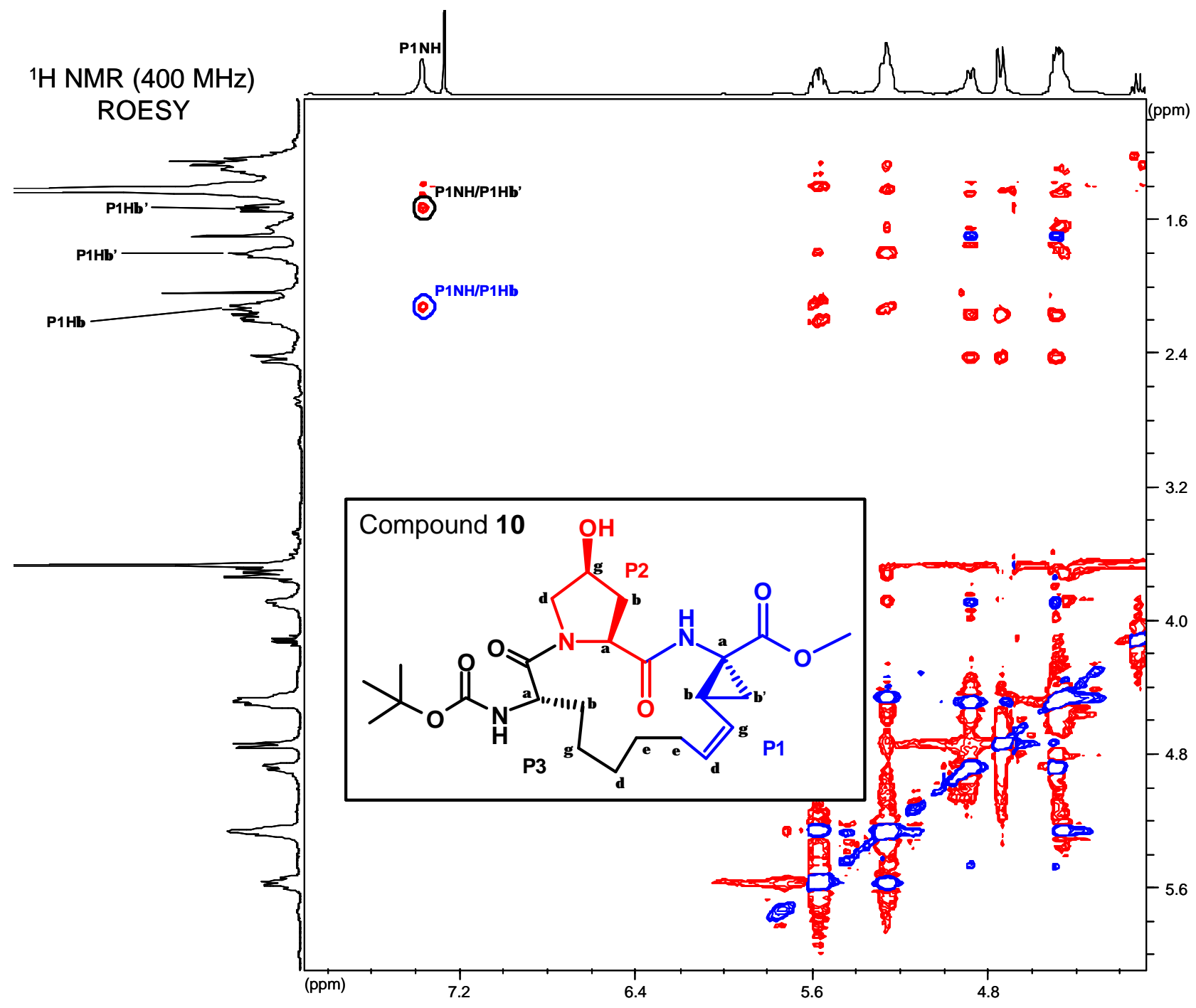

